\title{
The Burst Observer and Optical Transient Exploring System (BOOTES)
}

\author{
A.J. Castro-Tirado ${ }^{1,2}$, J. Soldán ${ }^{3}$, M. Bernas ${ }^{4}$, P. Páta ${ }^{4}$, T. Rezek ${ }^{3}$, R. Hudec ${ }^{3}$, T.M. Sanguino ${ }^{5}$, B. de la Morena ${ }^{5}$, \\ J.A. Berná ${ }^{6}$, J. Rodríguez ${ }^{7}$, A. Peña ${ }^{7}$, J. Gorosabel ${ }^{1}$, J.M. Más-Hesse ${ }^{1}$, and A. Giménez ${ }^{1,2}$ \\ 1 Laboratorio de Astrofísica Espacial y Física Fundamental (LAEFF-INTA), P.O. Box 50727, E-28080, Madrid, Spain \\ 2 Instituto de Astrofísica de Andalucía (IAA-CSIC), P.O. Box 03004, E-18080 Granada, Spain \\ 3 Astronomical Institute, Academy of Sciences of Czech Republic, 25165 Ondrejov, Czech Republic \\ ${ }^{4}$ Czech Technical University, Faculty of Electronic Engineering, Department of Radioelectronics, 16627 Prague, Czech Republic \\ 5 Centro de Experimentación de El Arenosillo (CEDEA-INTA), Mazagón, Huelva, Spain \\ 6 Departamento de Física, Ingeniería de Sistemas y Teoría de la Señal, Universidad de Alicante, Alicante, Spain \\ 7 Nuevas Tecnologías Observacionales (NTO), Pozuelo de Alarcón, Madrid, Spain
}

Received December 29, 1998; accepted April 9, 1999

\begin{abstract}
The Burst Observer and Optical Transient Exploring System (BOOTES) is considered as a part of the preparations for the ESA's satellite INTEGRAL, and is currently being developed in Spain, in collaboration with two Czech institutions. It makes use of two sets of wide-field cameras $240 \mathrm{kms}$ apart, and two robotic $0.3-\mathrm{m}$ telescopes. The first observing station (BOOTES-1) is located in Huelva (Spain) and the first light was obtained in July 1998. During the test phase, it has provided rapid follow-up observations for 5 GRBs detected by the BATSE aboard the CGRO. The system will fully operate in late 1999.
\end{abstract}

Key words: methods: observational — gamma-rays: bursts

\section{Introduction}

After years of search, the first optical counterparts to gamma-ray bursts (GRBs) have been found in 1997, beginning $3-20 \mathrm{hr}$ after the onset of the high energy events. A power-law decline with the flux $F \propto t^{\alpha}$ is usually observed, where $\alpha$ is in the range -1.1 to -2.0 . A review can be seen in Castro-Tirado (1999) and references therein. Under the assumption of the bursts being a repeating phenomenon, archival plates have been used in order to look for optical transient emission in the smallest GRB error boxes, and about 50 candidates have been identified so far, but most of them were rejected as they turned out to be plate defects (Hudec 1993).

Send offprint requests to: A.J. Castro-Tirado (ajct@laeff.esa.es)
Here we describe the Burst Observer and Optical Transient Exploring System (BOOTES), a set of instruments that is expected to contribute significantly to the understanding of GRBs and optical flashes.

\section{BOOTES: The concept}

\subsection{The Wide Field Cameras}

Commercial 50-mm wide-field lenses (Nikkor, Japan) are used, attached to two ST8 CCD cameras (Santa Barbara Instruments Group, USA). This resembles the Optical Transient Monitor (OTM) that is in operation at the Astronomical Institute in Ondrejov. Each pair of the BOOTES wide-field cameras is mounted atop a 0.3-m LX 200 Meade telescope (Meade, USA), allowing long integrations of a previously selected region. The four cameras monitor the same region of the sky, both in the $I$ and $V$-bands. The typical limiting magnitude is $V \sim 12$ for an integration time of $30 \mathrm{~s}$, and $V \sim 14$ for $300 \mathrm{~s}$ (see Table 1).

The first observing station (named BOOTES-1) is located at El Arenosillo (Huelva), a dark-sky site in Spain owned by the Instituto Nacional de Técnica Aerospacial (INTA). The other set will be placed at the Estación Experimental de La Mayora (Málaga), $240 \mathrm{~km}$ apart. The latter is run by the Consejo Superior de Investigaciones Científicas (CSIC).

The Wide Field Cameras make use of the Photomate 20 Signal Processor Board (Daimler-Benz Aerospace, Germany) which consists of two processors (see Bernas et al. 1998). The system is operated by commercial PCs. 
Table 1. Features of the BOOTES Wide Field Cameras

\begin{tabular}{|l|c|}
\hline \hline Lens & $50 \mathrm{~mm} \mathrm{@} \mathrm{f/1.2}$ \\
CCD & $1534 \times 1020$ pixels \\
field of view & $16^{\circ} \times 11^{\circ}$ \\
angular resolution & $0.63^{\prime}$ \\
limiting magnitude (300-s) & $V \sim 14$ \\
\hline
\end{tabular}

Table 2. Features of the BOOTES Robotic Telescope

\begin{tabular}{|l|c|}
\hline Telescope & $D=0.30 \mathrm{~m} @ f / 3.3$ \\
CCD & $1530 \times 1020$ pixels \\
field of view & $49^{\prime} \times 33^{\prime}$ \\
angular resolution & $1.9^{\prime \prime}$ \\
limiting magnitude $(60-\mathrm{s})$ & $R \sim 17$ \\
\hline
\end{tabular}

It is planned to work most of the time as discussed in Castro-Tirado et al. (1996), allowing to discriminate against flashing objects closer than one million kms thus ruling out satellite glints and other atmospheric and near-Earth events.

When information on a GRB position is obtained from the GCN (the GRB Coordinates Network, Barthelmy et al. 1998), the corresponding GRB error box is imaged by the cameras.

\subsection{The Robotic Telescope}

The Robotic Telescope is based on the Burst Alert Robotic Telescope (BART), that uses commercially available hardware components (Soldan et al. 1998). It is expected that the robotic telescope will perform rapid follow-up observations of events detected by BATSE, BeppoSAX, RossiXTE and future GRB detectors. The BOOTES telescope should be able to slew immediately and take deep frames at the GRB positions. Selected objects (variable stars, nearby galaxies, bright QSOs, etc.) will be regularly monitored, searching for flaring behaviour. The first telescope unit is already placed at the BOOTES-1 station, under a special enclosure which is opened automatically, according to weather conditions. The main features are shown in Table 2.

\section{Scientific objectives}

- The observation of the GRB error box simultaneously to the GRB occurrence ( $~ 6$ GRBs per year). The faint transient emission that has been detected few hours after the event, seems to be a consequence of the expanding remnant produced by the GRB. This provides information about the surrounding medium, but not about the burster itself. Although these optical counterparts are not brighter than 19 mag a few hours after the burst, transient emission simultaneous
Table 3. Quick follow-up GRB observations during 1998

\begin{tabular}{|l|c|c|c|}
\hline GRB & $\begin{array}{c}\text { Data taken } \\
\text { after }\end{array}$ & $\begin{array}{c}\text { Error box } \\
\text { coverage }\end{array}$ & $\begin{array}{c}\text { Limiting } \\
\text { mag. (I) }\end{array}$ \\
\hline 980808 & 66 -min & $95 \%$ & 13 \\
980810 & 125 -min & $100 \%$ & 13 \\
981009 & 255 -min & $100 \%$ & 13 \\
981203 & $35-$ min & $100 \%$ & 13 \\
981205 & $30-$ min & $100 \%$ & 13 \\
\hline
\end{tabular}

to the event is expected to be more intense. In fact, an extrapolation of the $\gamma$-ray power-law spectrum indicates that the simultaneous optical flash should lie in the range $10-15 \mathrm{mag}$, depending on the burst intensity. Current theories predict that there should be an optical flash reaching a red broad-band magnitude $R \sim 10$ or brighter, as has been seen for the first time in GRB 990123 (Akerlof et al. 1999).

- The detection of optical flashes of cosmic origin, that could be unrelated to GRBs and would be a different astrophysical phenomenon (perhaps associated to QSOs/AGNs). In fact some GRB models predict that there should be a large number of bursting sources in which only transient X-ray/optical emission should be observed, but no $\gamma$-ray emission. The latter would be confined in a jet-like structure and pointing towards us only in a few cases.

- The observation of the sky in the $I$ and $V$ filters, as a part of the preparations for the ESA's satellite project INTEGRAL (the International Gamma-Ray Laboratory), in which Spain and the Czech Republic are involved with the Optical Monitoring Camera (OMC, Más-Hesse et al. 1998). The preparation includes test of technologies, data processing, ground based observational network, etc.

- The monitoring of several objects (bright AGNs/QSOs, old GRB positions, etc.) looking for recurrent transient optical emission arising from these sources (Hudec et al. 1996). There are hints that sudden and rapid flares occur. This will be achieved by means of the 0.3-m BOOTES telescope.

\section{Current status}

The BOOTES-1 first light took place on 1 July 1998, and the quick follow-up GRB observations performed so far are summarized in Table 3. The two stations (BOOTES-1 and -2) will fully operate in Dec. 1999. Additional information can be seen at http://www.laeff.esa.es/ ajct/BOOTES/bootes98.html

Acknowledgements. We are very grateful for the support given by the Space Sciences Division (DCE) at INTA, through the project IGE 4900506. We thank J. Maíz, R. Morales, M. Santos-Lleó and J. Torres for very fruitful conversations, M. Iríbar, J. Martín-Francía, F. Souvrier and J.M. Vilaplana 
for their hospitality and help at El Arenosillo, and the anonymous referee for useful suggestions. We are also indebted to the GCN team (in particular to S. Barthelmy) for rapidly distributing the GRB positions obtained by BATSE. The Czech contribution is supported by the Ministry of Education and Youth of the Czech Republic, projects ES02 and ES36.

\section{References}

Akerlof C., et al., 1999, Nat 398, 400

Barthelmy S., et al., 1998, AIP Conf. Proc. 428, Meegan C.A., Preece R.D., Koshut T.M. (eds.), p. 99

Bernas M., Pata P., Hudec R., Soldán J., Rezek T., Castro-
Tirado A.J., 1998, AIP Conf. Proc. 428, Meegan C.A., Preece R.D., Koshut T.M. (eds.), p. 864

Castro-Tirado A.J., Hudec R., Soldan J., 1996, AIP Conf. Proc. 384, Kouveliotou C., Briggs M.F., Fishman G.J. (eds.), p. 814

Castro-Tirado A.J., 1999, A\&SS (in press) (astro-ph/9903187)

Hudec R., 1993, Astrophys. Lett. Comm. 28, 359

Hudec R., et al., 1996, ASP Conf. Ser. 110, Miller H.R., Webb, Noble J.C. (eds.), p. 129

Más-Hesse J.M., et al., 1998, Phys. Scr. T77, 44

Soldán J., Hudec R., Nemcek M., Rezek T., 1998, AIP Conf. Proc. 428, Meegan C., Preece R.D., Koshut T.M. (eds.), p. 855 